\title{
Blind User Response to a Navigational System to Assist Blind People Using Active RFID and QR-Code
}

\author{
Saleh Alghamdi \\ School of Computer Science \& IT \\ RMIT University \\ saleh,alghamdi@rmit.edu.au
}

\author{
Ron van Schyndel \\ School of Computer Science \& IT \\ RMIT University \\ ron.vanschyndel@rmit.edu.au
}

\author{
Margaret Hamilton \\ School of Computer Science \& IT \\ RMIT University \\ margaret.hamilton@rmit.edu.au
}

\begin{abstract}
In previous papers, we presented a navigational system for use by the blind and people with low vision. The system was built upon two technologies, Radio Frequency Identification (RFID) tags and Quick Response (QR) Codes. The system has been tested by sighted people and it has reached a high level of satisfactory results to identify the current position of the user with an accuracy of less than two meters in most cases, and to safely guide users to a desired destination. The new positioning technique is based on line-of-sight QR code detection, and non-line-of-sight signal attenuation of active RFID tags using a wearable reader. The aim of this paper is to present some user feedback of the system from the blind. One of the significant outcomes of the usability test on blind participants is that the system has to work in an integrated manner in order to achieve the aspirations of the users.
\end{abstract}

\section{Categories and Subject Descriptors}

H.5.2 [User Interfaces]: Evaluation / methodology, Voice I/O

\section{General Terms}

Management, Measurement, Documentation, Reliability, Experimentation, Human Factors, Languages, Verification

\section{Keywords}

Positioning, Navigation, RFID, QR-Code and Blind Assistance.

\section{INTRODUCTION}

One of the most significant rehabilitation steps for people who have recently become blind, or have low vision, is for such people to regain their ability to navigate and react to their immediate environment independently and therefore to feel that they are in control again [1]. This is what motivated the authors to develop a system using both line-of-sight and non-line-of-sight technologies to give the user both passive and active feedback about their position.
Permission to make digital or hard copies of all or part of this work for personal or classroom use is granted without fee provided that copies are not made or distributed for profit or commercial advantage and that copies bear this notice and the full citation on the first page. To copy otherwise, to republish, to post on servers or to redistribute to lists, requires prior specific permission and/or a fee. REHAB 2014, May 20-23, Oldenburg, Germany Copyright (c) 2014 ICST 978-1-63190-011-2 DOI 10.4108/icst.pervasivehealth.2014.255354
Our aims of the tests in this paper are to discover the limitations of the system from the perspective of blind users and to evaluate the satisfaction of the real users regarding the developing navigational system.

The whole scenario of the larger system is summarized in Fig. 1. The cubes represent the hardware components which are active RFID, QR-code and Kinect and the rounded rectangles represent the main services algorithms of the system. For the work of this paper, the Kinect component was not available or used.

The navigation process follows three steps: Firstly, the system indicates the initial position of the user as a start point using our RFID algorithm which is based on the technique of attenuation control. Secondly, the user is recommended to scan surrounding area using a camera to detect QR-code using built-in QR-code reader application. Finally, the navigation application determines

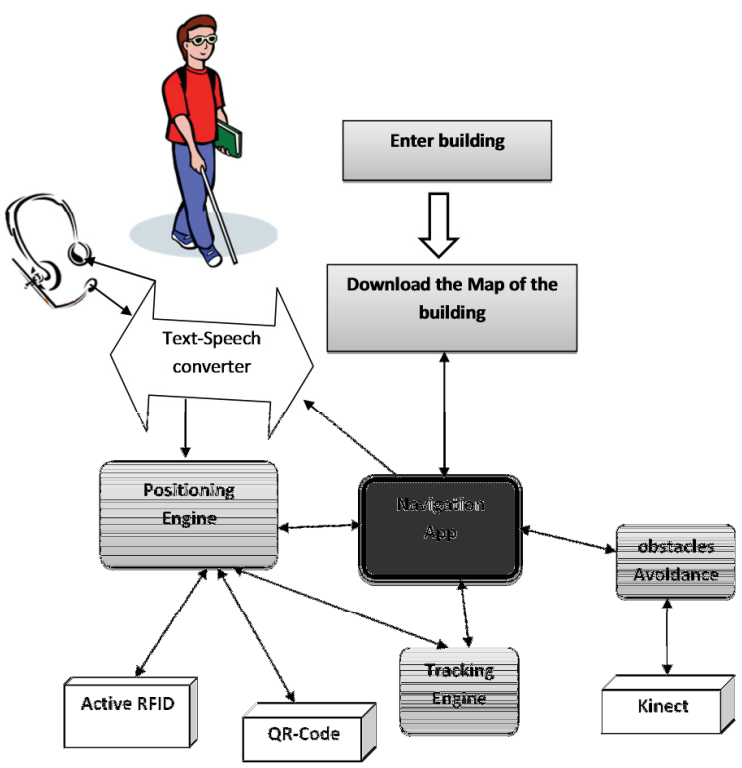

Figure 1. The Scenario of The Proposed Navigational System.

the more preferable route to the user. Then it starts providing voice directions to the user. The route calculation is based on the Dijkstra algorithm which indicates the shortest path between two points (the current position and the destination).

We describe the system in detail in $[11,12]$ and performed initial testing in our office area as shown in Fig. 2. 


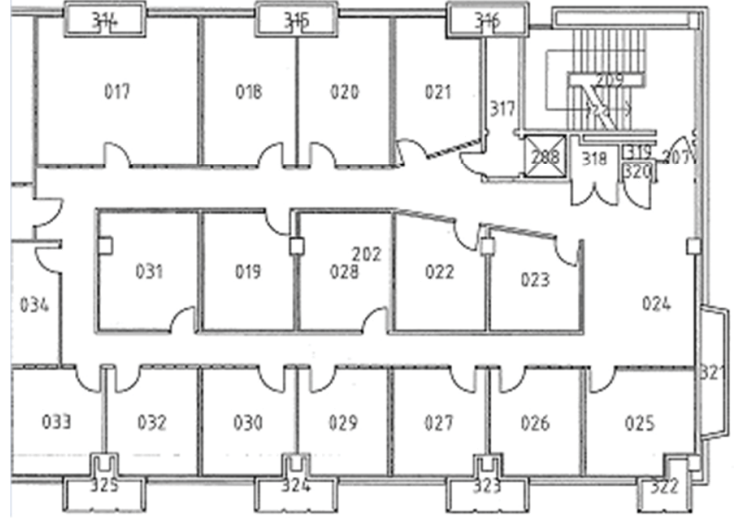

Figure 2. A typical office layout.

The system created has achieved highly successful results to identify the current position of the user and to guide that user to various destinations within the domain shown in Fig 3. Therefore, the authors were enthusiastic to apply the system on real participants who are blind people which represents the second stage of our usability testing. The participants were eight male students between seventeen and twenty years of age. The path had been established in an indoor school gymnasium, QR codes printed on A4 size sheets of paper and RFID tags were attached on pillars at various points in the room as shown in Fig. 3. The distance between each pair of pillars was measured and the system has been adapted with that environment. All tests were conducted during a school day in a real life crowded scenario.

\section{RELATED WORK}

Over the years, many navigational technologies have been developed to aid the blind and people with low vision [2-9]. Much research has been conducted into computer scene-understanding at both a low- and high-level, using many individual modalities such as the navigation tool proposed by Mihajlik [10] which is based on connecting sound generation to a navigation system, using a Digital Signal Processor, and using ultrasonic echolocation which has been applied successfully in mobile robots with a 3D sound generation technique. Another attempt presented

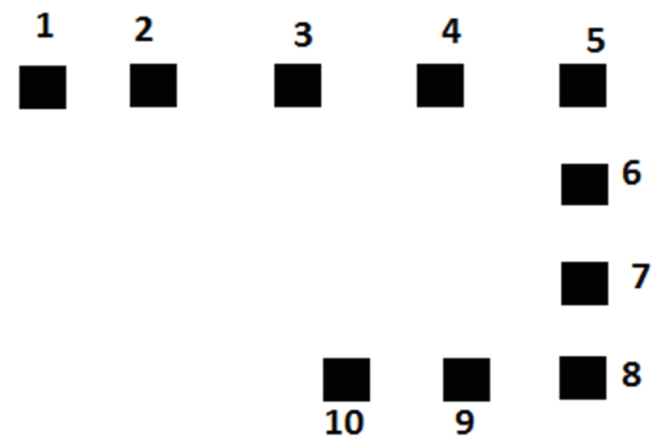

Figure 3. The positions of pillars where RFID tags and QR-Codes were distributed and used for usability test purposes as the second stage of testing.

in [2] is Electronic Travel Aids (ETAs). An ETA is similar to Mihajlik's device in that both use ultrasonic waves to detect obstacles but ETAs aim to identify objects specifically for visually impaired users. Later research [5] investigated a vision sensor camera to capture images and then process these images and convert them to sound. Almost all this research used a gray level technique to identify objects in images, the 'NAVI' system [5] being a well-known example of this approach.

\section{PLANNING THE TEST}

The requests from participants have been designed from considering a simple task such as walking from point 2 toward point 3 along the same line as shown in Fig. 3, to more complex tasks, for instance, the last task from point 9 to point 2. This concept has been used to give participants the chance to become more familiar with the system. Therefore, we believe that training on the system is a necessary requirement for new users to achieve the full benefit of the system.

Requests To participants:

- $\quad$ Identify the destination. As shown in Fig 4a, low vision people may be able to determine the destination by clicking the "Go to" button, but voice recognition feature has been added to the system to assist blind people to identify their desired destination.

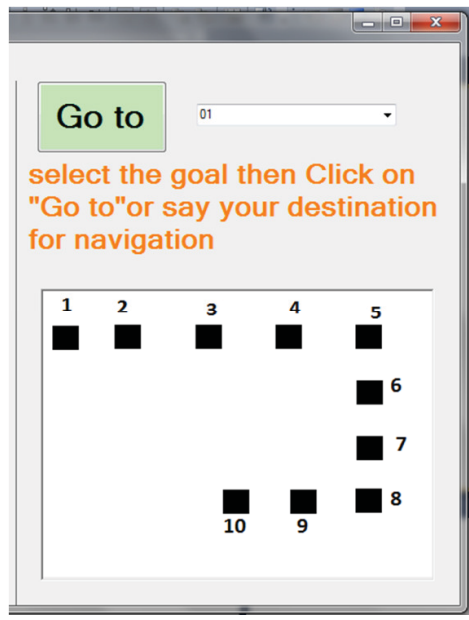

A

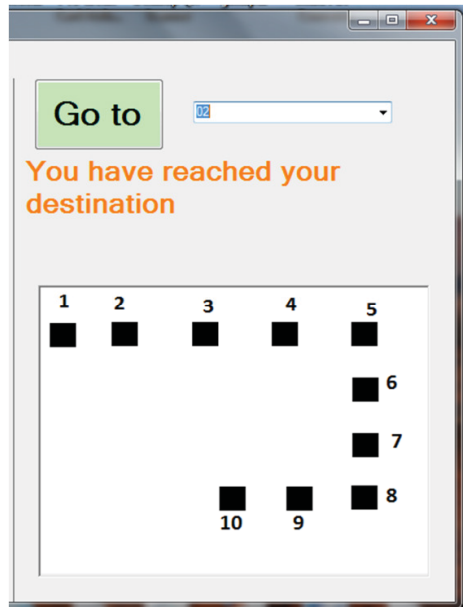

B

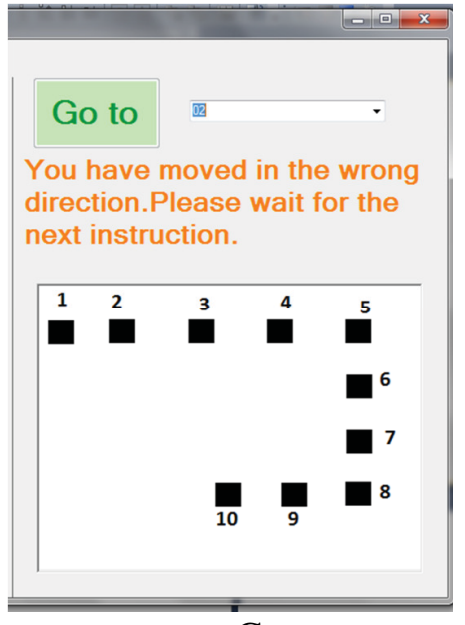

C

Figure 4. Interface of Navigation Program, where A represents the start interface, B represents the final stage when the user reaches the destination successfully and $\mathrm{C}$ represents an error detection feature. 
- $\quad$ Make an error. Each participant was asked to commit a deliberate error such as turn left instead of follow the instruction of the system and turn right. The purpose of that was to evaluate Error Detection feature as shown in Fig. 4c. In addition, to test the ability of the system to recalculate an alternative path to guide the user from his current incorrect location back to his target destination successfully.

- $\quad$ Recognise when you have arrived. By seeing/hearing the message of Fig $4 \mathrm{~b}$

- $\quad$ Applying the QR-Code navigation system alone. Each participant was been asked to walk to five different destinations using the QR-Code alone by pointing the camera in the right general direction.

- $\quad$ Applying the RFID navigation system alone. Each participant was asked to do the test again using RFID technology, where pointing was unnecessary, but location was slightly more error-prone.

\section{REPORTING RESULTS}

After participants completed the requested navigational tasks, they were asked to answer the following questions:

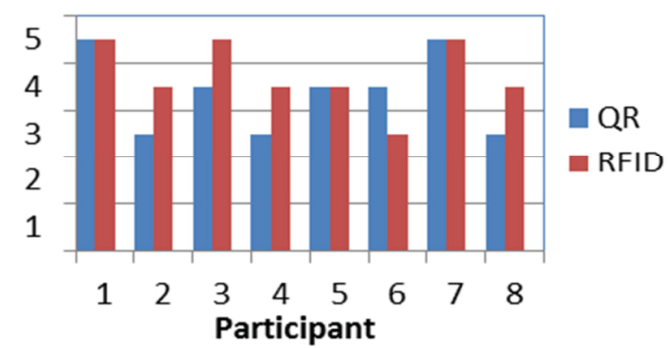

Figure 5. Q1 - Do you feel that you were guided well to reach the destinations? Grading 1 (Unsatisfactory) to 5 (Excellent)

The average confidence of using the navigation system with QRCodes wass 3.875 out of 5 to reach destinations while using RFID system the average of the trust was 4.25 out of 5 as shown in Fig. 5. Half numbers of participants were more trusting of the RFID system to help them reach their destinations. Three participants trustedthe RFID and QR-Code systems equally and only one participant preferred QR-Code over RFID system to guide him to his destinations.

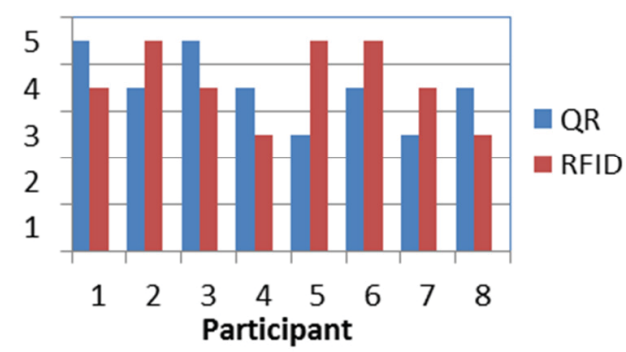

Figure 6. Q2 - Do you believe you reached each waypoint on time according to the program (1=unsatisfactory, 5=very satisfied)?
The system estimates the required time to reach each waypoint in a trip to track the user for error detection purposes. The required time is calculated based on a 'standard' blind walking speed of approximately 1 meter per second. The average of satisfaction for the required time calculation method was similar for QR-Code and RFID where both of them were around 4 out of 5 as shown in Fig.6.

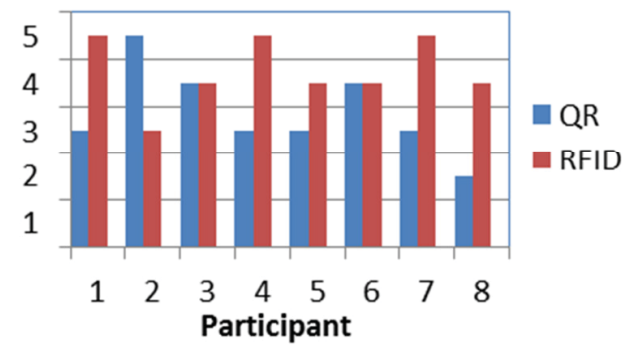

Figure 7. Q3 - When the program told you there was an error, did you understand what the system asked you to do next ( $1=$ not at all, $5=$ very much $)$ ?

This question belongs to an error detection feature plus enabled us to study to which degree the participants believed that the systems were able to recalculate an alternative path to guide them to the destination. As shown in Fig. 7, the satisfaction of participants regarding the QR-Code system was 3.375 out of 5 and the average level of satisfaction increased with the RFID system to 4.25 of 5 for error detection and rerouting of participants to their destinations successfully.

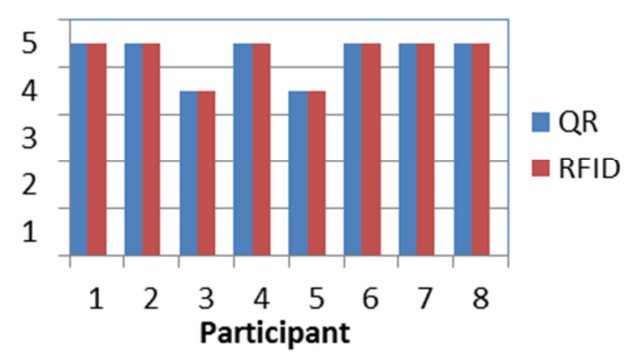

Figure 8. Q4 - Do you feel you got more confident using the system over time (1=not at all, $5=$ very much)?

Almost every participant gave a score of 4 or 5 out of 5 regarding the benefit of training on the system. All participants believed that training is required and helpful for both systems so they gave the same scores for both systems regarding this question.

$$
\text { Q5 - Do you suggest any improvement? }
$$

Most suggestions were regarding voice instructions in terms of language and volume level. Around one-third of the participants preferred to hear Arabic instructions. Also three quarters of participants found it difficult to hear the instructions because of the background noise in the school.

The instructions were the same for both systems so all participants gave the same score for their levels of satisfaction for the way the instructions were delivered where the average level of satisfaction was only 2 out of 5 for both systems as shown in Fig. 9 . 


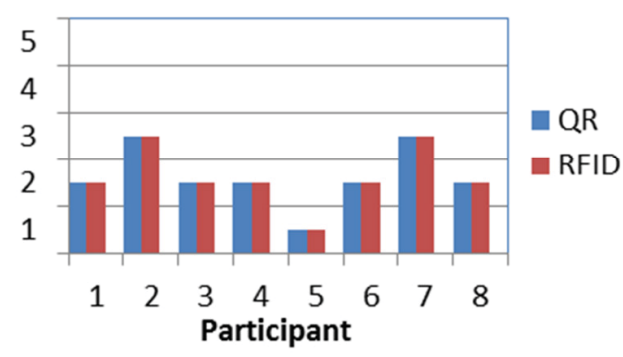

Figure 9. Q6 - Do you believe that the instructions which the system gives need to be improved (1=Happy with it, $5=$ Much improvement needed)?

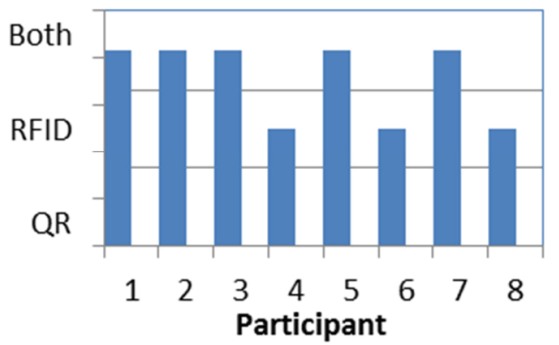

Figure 10. Q7 - With which of the two systems are you more comfortable? the QR code system, or the RFID system? Or do you prefer both of them? Why?

Fig. 10 shows that three of the participants preferred to use the RFID navigation system. In contrast, all other participants believed that the integrated system (RFID + QR-Code) together was more beneficial and useful for them.

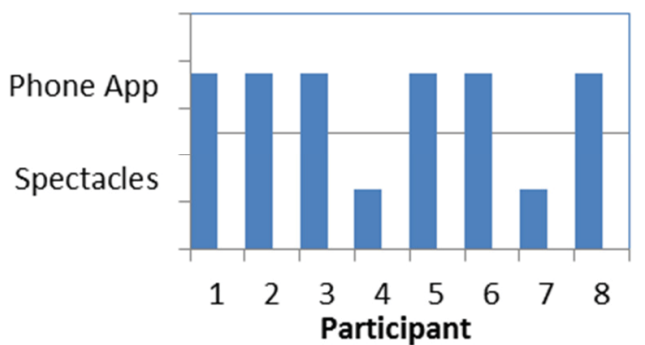

Figure 11. Q8 - If we turned this system into a product that you can buy, would you prefer it as a mobile phone app or as spectacles that can speak into your ears?

As shown in Fig. 11, two participants hoped to use this navigation system and receive the navigational instructions via spectacle or headphone. The other participants preferred to have this navigation system installed as an application on their smart phones.

\section{CONCLUSION}

We presented a user test of a designed navigational system based on active RFID technology and QR-Codes. The system has been tested in two stages, firstly by sighted people for debugging purposes and secondly by blind people for usability. Regarding the results of the usability test we found that we have to integrate RFID and QR-Codes to reach the highest levels of satisfaction for the real users of the system who are blind people. Because the active RFID system works independently and its accuracy is less than two meters, and range is up to 70 meters, to get higher precise positioning information the user needs to start scanning in that two meter area using QR-Codes which are very difficult for the blind to detect without the assistance of RFID.

\section{REFERENCES}

[1] http://www.sciencedaily.com/releases/2007/02/07021622152 2.htm, accessed 10 April , 2014.

[2] G. Balakrishnan, G. Sainarayanan, R. Nagarajan, S. Yaacob, "Stereo Image To Stereo Sound Methods For Vision Based ETA" Computers, Communications \& Signal Processing with Special Track on Biomedical Engineering, (CCSP), pp.193196, 14-16 Nov. 2005.

[3] D. J. Calder, "Travel Aids For The Blind - The Digital Ecosystem Solution", 7th IEEE International Conference on Industrial Informatics (INDIN), pp. 149-154., 2009

[4] M. H. Choudhury, D. Aguerrevere, A. B. Barreto, "A PocketPC Based Navigational Aid For Blind Individuals" in IEEE Virtual Environments, Human-Computer Interfaces and Measurement Systems (VECIMS), pp. 43-48, 2004

[5] R. Nagarajan, G. Sainarayanan, S. Yacoob, R. Porle, "An Improved Object Identification For NAVI," in IEEE Region 10 Conference (TENCON), 2004, pp.455-458 Vol. 1.

[6] R. Nagarajan, S. Yaacob, G. Sainarayanan, "Fuzzy Clustering In Vision Recognition Applied In NAVI," Fuzzy Information Processing Society, 2002. Proceedings. NAFIPS. 2002 Annual Meeting of the North American, pp. 261-266, 2002

[7] R. Nagarajan, S. Yaacob, G. Sainarayanan, "Role Of Object Identification In Sonification System For Visually Impaired", TENCON, 2003. Conference on Convergent Technologies for Asia-Pacific Region, pp. 735-739 Vol.2, 15-17 Oct. 2003.

[8] L. Xia, C. Chen and J. K. Aggarwal, "Human Detection Using Depth Information by Kinect", IEEE Computer Society Conference on Computer Vision and Pattern Recognition Workshops (CVPRW), pp. 15-22,2011,

[9] F. Wong, R. Nagarajan, S. Yaacob, "Application Of Stereovision In A Navigation Aid For Blind People" Information, Communications and Signal Processing, 2003 and the Fourth Pacific Rim Conference on Multimedia. Proceedings of the 2003 Joint Conference of the Fourth International Conference, vol.2, pp. 734- 737, 2003

[10] P. Mihajlik, M. Guttermuth, K. Seres, P. Tatai, "DSP-based Ultrasonic Navigation Aid For The Blind" in Proceedings of the 18th IEEE Conference on Instrumentation and Measurement Technology, (IMTC), pp. 1535-1540, 2001

[11] Saleh Alghamdi, Ron van Schyndel, Ibrahim Khalil, "Accurate positioning using long range active RFID technology to assist visually impaired people", in Journal of Network and Computer Applications, Elsevier Preprint, Accepted Nov 2013, Preprint DOI: 10.1016/j.jnca.2013.10.015

[12] Saleh Alghamdi, Ron van Schyndel, Ahmed Alahmadi, "Indoor Navigational Aid Using Active RFID and QRCode For sighted and Blind People", Eighth International Conference on Intelligent Sensors, Sensor Networks and Information Processing (ISSNIP), Melbourne, Australia, April 2013, pp18-22 